\title{
Exploratory investigation of nucleon-nucleon interactions using Euclidean Monte Carlo simulations
}

\author{
Istvan Montvay ${ }^{a, b}$ and Carsten Urbach $^{b}$ \\ ${ }^{a}$ Deutsches Elektronen-Synchrotron DESY, Notkestr. 85, D-22603 Hamburg, Germany \\ ${ }^{b}$ HISKP (Theorie), Universität Bonn, Nussallee 14-16, D-53115 Bonn, Germany
}

May, 2011

\begin{abstract}
We present an exploratory study of chiral effective theories of nuclei with methods adopted from lattice quantum chromodynamics (QCD). We show that the simulations in the Euclidean path integral approach are feasible and that we can determine the energy of the two nucleon state. This opens up the possibility to determine in future simulations nucleon phase shifts by varying the parameters and the simulated volumes. The physical cut-off of the theory is realised by blocking of the lattice fields. By keeping the block size fixed in physical units the lattice cut-off (i.e. the lattice spacing) can be freely changed. This offers an effective way for controlling lattice artefacts.
\end{abstract}




\section{Introduction}

Quantum Chromodynamics (QCD) is the nowadays accepted theory of strong interactions in terms of the fundamental quark and gluon degrees of freedom. Also, if one is interested in nuclear physics, QCD is the relevant theory to calculate for instance binding energies of nuclei from first principles. However, QCD is strongly interacting at low energies and hence, non-perturbative methods are needed in order to study quantities as, for instance, nuclear binding energies.

The method of choice for ab initio, non-perturbative investigations of QCD is provided by lattice QCD. Even if this field was facing tremendous progress over the last few years, the computation of nuclear binding energies for say $A>2$ is still out of reach. But it is also not clear whether those quantities need to be computed in the fundamental theory, because the relevant degrees of freedom are effectively given by hadrons described in a chiral effective theory [1,2], and not by quarks and gluons in QCD. And again, lattice methods can be used in order to investigate these chiral effective theories from first principles, for a review see ref. [3]. For an overview over the various available approaches and methods to nuclear physics using chiral effective theories we refer to recent review articles [4, 5].

A lattice approach to nuclear physics was presented in a row of papers by Borasay and collaborators [6-12]. The authors of these publications take the approach of simulating the chiral effective theory on a discrete space-time lattice. Starting point for these simulations is the non-relativistic effective theory. As a consequence, Quantum Monte-Carlo methods based on Fock states [13] are used for the numerical simulations. The lattice regularisation is utilised as the physical cut-off in the theory, hence it cannot be removed. It naturally takes values of $a \sim 2 \mathrm{fm}$. Lattice artifacts are reduced perturbatively by adding counter-terms to the simulated action. Such large values of the lattice spacings allow for simulations with $L=8$ lattice points at most in each of the spatial directions. This is important, as in the simulations a sign problem is apparent, which is controllable for small $L$, but severe for large $L$.

Apart from the fact that QCD and the chiral effective field theory (ChET) are rather different, also the approach taken in these publications differs fundamentally from the methods applied in LQCD simulations. In LQCD the path integral in Euclidean space-time is directly simulated using appropriate Markov chain Monte Carlo methods. Simulations are performed in a finite volume with discretised space-time, with the inverse lattice spacing serving as a momentum cut-off. Renormalisaton is then performed by properly removing the cut-off in the continuum limit when the lattice spacing tends to zero $(a \rightarrow 0)$. At this point the fundamental difference between QCD and ChET becomes evident: LQCD has a non-trivial continuum limit and hence the cut-off can be completely removed. In ChET the 
cut-off cannot be removed - in fact it has a physical relevance representing the energy scale where the basic degrees of freedom (pions and nucleons) become inappropriate for describing physics. In a perturbative framework the necessity of a finite cut-off is implied by the non-renormalisability of the theory. In a non-perturbative formulation ChET belongs to the general class of Yukawa-type theories with fermion-boson interactions. (In a four-fermion theory, for instance, the boson in the Yukawa interaction is generated dynamically.) Yukawatheories are expected to have only a trivial (non-interacting) continuum limit, therefore the cut-off is necessary for maintaining a non-zero interaction. (For an introduction in lattice Yukawa models see Chapter 6 in [14].) The finite value of the cut-off also corresponds to the non-zero size of pions and nucleons below which distance scale obviously some other description is required (namely, with quarks and gluons in terms of QCD).

In the continuum limit of LQCD all symmetries of the continuum theory broken by discretisation, including Lorentz symmetry, are restored and lattice artifacts in all quantities are removed. Performing the continuum limit requires an extrapolation to vanishing value of the lattice spacing $a \rightarrow 0$ and therefore the simulations have to take place in ranges of $a$ which allow such an extrapolation in a controlled way. It is not a priori clear how small values of the lattice spacing are required for this procedure, and eventually one will only find it out empirically.

The so called Symanzik effective theory [15-17] allows for a better understanding which lattice artifacts one has to expect. For sufficiently small values of $a$ LQCD can be described by an effective local action [18]

$$
S_{\mathrm{eff}}=S_{0}+a S_{1}+a^{2} S_{2}+\ldots
$$

where $S_{0}$ is the continuum QCD action and the additional terms $S_{k}$ for $k>0$ are to be interpreted as operator insertions in the continuum theory

$$
S_{k}=\int d^{4} x \mathcal{L}_{k},
$$

with $\mathcal{L}_{k}$ being combinations of local composite fields with mass dimension $4+k$. The list of possible fields is constrained by the symmetries. Similarly one can write for a local gauge invariant composite field $\phi(x)$, where we neglect mixing under renormalisation for simplicity,

$$
\phi_{\text {eff }}(x)=\phi_{0}(x)+a \phi_{1}(x)+a^{2} \phi_{2}(x)+\ldots .
$$

The fields $\phi_{k}$ are again linear combinations of local fields with appropriate symmetries and dimension. Without discussing all the details - which can be found in ref. [18] - one finds 
that a connected $n$-point function on the lattice to leading order in the lattice spacing reads

$$
\begin{aligned}
& G_{n}\left(x_{1}, \ldots, x_{n}\right)=\left\langle\phi_{0}\left(x_{1}\right) \cdots \phi_{0}\left(x_{n}\right)\right\rangle^{\mathrm{cont}} \\
& \quad-a \int d^{4} y\left\langle\phi_{0}\left(x_{0}\right) \cdots \phi_{0}\left(x_{n}\right) \mathcal{L}_{1}(y)\right\rangle^{\mathrm{cont}} \\
& \quad+a \sum_{k=1}^{n}\left\langle\phi_{0}\left(x_{1}\right) \cdots \phi_{1}\left(x_{k}\right) \cdots \phi_{0}\left(x_{n}\right)\right\rangle^{\mathrm{cont}} \\
& \quad+\ldots
\end{aligned}
$$

From this expression it should be clear that first of all the value of $a$ should be small enough to neglect all higher order terms in the lattice spacing. In particular, on the right hand side of eq. (3) there should be only combinations with $a\langle\ldots\rangle \ll 1$. This is also the reason why LQCD investigations with heavy quarks appear to be difficult since then $a m_{q}$ can easily become of order 1 and can lead to significant lattice artifacts. Of course, all terms on the right hand side of eq. (3) come with unknown coefficients, so also with $a m_{q} \approx 1$ large lattice artifacts are not automatically to be expected.

The so called Symanzik expansion can also be used to systematically remove lattice artifacts from the theory. This requires to add counter terms to the lattice action with coefficients that need to be determined non-perturbatively. Improving the action is, however, not always enough. There are observables that require operator specific improvement with coefficients that again need to be determined non-perturbatively.

Comparing the lattice QCD approach and the lattice approach to chiral effective theories from refs. 6 12 rises an immediate question, namely how large are lattice artifacts in the lattice simulations of the chiral effective theory and whether they are will controlled. This question is hard to answer since the continuum limit $a \rightarrow 0$ cannot be performed due to the physical interpretation of the cut-off. Moreover, a variation of the cut-off is for technical reasons difficult, but a variation in a reasonable range is desirable.

This is why we take a different approach to lattice simulations of these chiral effective theories, which we shall present in this paper. It consists of using the fully relativistic path integral formulation of the chiral effective theory in Euclidean space-time, which can be simulated by means of Markov chain Monte Carlo methods. The physical cut-off of the theory is implemented by block-field methods. The sizes of the blocks represent the hadron sizes. These have to be kept fixed if the lattice spacing is changed, for instance, keeping $R \cdot M$ fixed where $R$ denotes the block size and $M$ is a physical mass, say the nucleon mass. Separating the lattice cut-off from the physical cut-off allows to change the lattice cut-off (i.e. the lattice spacing) by keeping the physical content of the theory unchanged. On the basis of the Symanzik effective theory it has to be expected that by making the lattice spacing sufficiently small the lattice artifacts, for instance, Lorentz-symmetry breaking can 
be suppressed. One can also imagine to reach in the limit $a \rightarrow 0$ a non-trivial continuum limit describing an interacting non-local quantum field theory. Strictly speaking, the existence of such a continuum limit is not known at present - similarly to the existence of the continuum limit of many other lattice quantum field theories.

A substantial simplification in fermionic lattice quantum field theories is the so called quenched approximation which corresponds to omitting closed fermion loops. Since both neutron and proton are heavy, we do not expect that nucleon-anti-nucleon loops play a significant role and hence perform the simulations in the quenched approximation. Later on one can, of course, perform simulations in the full theory, removing this approximation.

Another possible simplification is to work within a non-relativistic approximation - instead of in a relativistic quantum field theory as we are doing here. One big advantage of the non-relativistic formulation might be that the rest masses of the nuclei do not appear in the theory and binding energies are computed directly, whereas in our relativistic approach binding energies are sub-percent effects as compared to the energy levels we determine. This is one point we shall address in a forthcoming publication.

The authors of refs. [19 21] follow a similar approach to the one presented here, but applied to a Yukawa model with one scalar field. Previous investigations of Yukawa models in the Euclidean path integral formulation have been performed in the context of upper and lower limits of masses in the electroweak theory, see for instance refs. [22, 23].

In this paper we shall discuss our method and show evidence for its applicability. Physical results are planned to be presented in forthcoming publications. In the following section we discuss our lattice action followed by details of the numerical methods we apply. We shall close with presenting some simulation results and give a conclusion and outlook.

\section{Lattice actions}

Let us start by introducing our notations. The nucleon fields are described by a pair of Grassmann variables and are denoted by

$$
\psi_{\alpha x}, \quad \bar{\psi}_{\alpha x} \quad(\alpha=1,2)
$$

where $\alpha$ is the isospin doublet index and $x$ denotes the lattice sites. The Dirac-index of the nucleon field is not displayed here and the isospin index of the nucleon field will also be omitted in most formulae. The real boson fields are

$$
\phi_{a x}^{(\pi)}, \quad \phi_{x}^{(0)}, \quad \phi_{a x}^{(1)} \quad(a=1,2,3)
$$

with the triplet isospin index $a$. Here $\phi^{(\pi)}$ stands for the pion and $\phi^{(0)}$ and $\phi^{(1)}$ are HubbardStratanovich auxiliary boson fields with isospin zero and one, respectively. 
These latter are used to describe four-nucleon interactions which in the Euclidean lattice action have the following form:

$$
S_{\mathrm{NA}}=\sum_{x}\left\{\phi_{x}^{(0)} \phi_{x}^{(0)}+\phi_{a x}^{(1)} \phi_{a x}^{(1)}+C_{0} \phi_{x}^{(0)}\left(\bar{\psi}_{x} \psi_{x}\right)+C_{1} \phi_{a x}^{(1)}\left(\bar{\psi}_{x} \tau_{a} \psi_{x}\right)\right\}
$$

where $\tau_{a},(a=1,2,3)$ are Pauli-matrices for isospin and a summation over repeated indices $a$ is understood. The four-nucleon interactions are obtained after integrating over the auxiliary fields according to

$$
\int_{-\infty}^{\infty} d \phi \exp \left\{-\phi^{2}-C \phi(\bar{\psi} \psi)\right\}=\sqrt{\pi} \exp \left\{\frac{C^{2}}{4}(\bar{\psi} \psi)^{2}\right\} .
$$

Our choice of the pion-nucleon interaction corresponds to the lattice discretisation of the continuum interaction $\partial_{\mu} \pi_{a}\left(\bar{\psi} \gamma_{5} \gamma_{\mu} \tau_{a} \psi\right)$ and is given as

$$
S_{\mathrm{N} \pi}=\sum_{x}\left\{\frac{C_{\pi}}{2}\left(\phi_{a x+\hat{\mu}}^{(\pi)}-\phi_{a x-\hat{\mu}}^{(\pi)}\right)\left(\bar{\psi}_{x} \gamma_{5} \gamma_{\mu} \tau_{a} \psi_{x}\right)\right\},
$$

where $\hat{\mu}$ denotes, as usual, the unit vector on the lattice in direction $\mu(\mu=1,2,3,4)$. (Similarly to isospin index $a$, over repeated direction index $\mu$ a summation is implied.) The above expression corresponds to a particularly simple discretisation of the derivative of the pion field which can, of course, be chosen differently.

The parameters of the lattice action are always dimensionless. The connection to the (eventually) dimensionful parameters in continuum formulations is established with the multiplication by an appropriate power of a mass parameter.

Let us illustrate this on the example of the four-nucleon interactions in (6)-(7)). The (bare) nucleon field $\psi_{x}$ is related to the (bare) continuum nucleon field by $\psi_{x}=a^{3 / 2} \psi_{x}^{\text {cont }}$ (and similarly for $\left.\bar{\psi}_{x}\right)$. The relation for the auxiliary scalar fields is given by $\phi_{x}=a^{2} \phi_{x}^{\text {cont }}$. This implies that the four-nucleon couplings $C_{0,1}$ are related to their continuum counterparts by $C_{0,1}=a^{-1} C_{0,1}^{\text {cont }}=\left(m_{\mathrm{N}} C_{0,1}^{\text {cont }}\right) /\left(a m_{\mathrm{N}}\right)$. Here, $m_{\mathrm{N}} C_{0,1}^{\text {cont }}$ is again dimensionless and independent of the lattice spacing $a$. In the following we always work in lattice units. The connection to the (eventually) dimensionful parameters in continuum formulations is established by multiplication with an appropriate power of a mass parameter and/or a power of the lattice spacing $a$. Note that in Ref. [7] the coefficients appear quadratically in the action.

In addition to the interaction terms we also need a kinetic term for the nucleon which we take to be the Wilson fermion lattice action [24]:

$$
S_{\mathrm{N}}=\sum_{x}\left\{\left(\bar{\psi}_{x} \psi_{x}\right)-\kappa_{\mathrm{N}} \sum_{\mu= \pm 1}^{\mu= \pm 4}\left(\bar{\psi}_{x+\hat{\mu}}\left[1+\gamma_{\mu}\right] \psi_{x}\right)\right\} .
$$


Here $\kappa_{\mathrm{N}}$ is the hopping parameter defining the nucleon mass and the convention $\gamma_{-\mu}=-\gamma_{\mu}$ is followed. For the pion field, besides the kinetic term, also a self-interaction term is allowed, hence we define the pion part of the action by

$$
S_{\pi}=\sum_{x}\left\{-2 \kappa_{\pi} \sum_{\mu=1}^{\mu=4} \phi_{a x}^{(\pi)} \phi_{a x+\hat{\mu}}^{(\pi)}+\lambda\left[\phi_{a x}^{(\pi)} \phi_{a x}^{(\pi)}-1\right]^{2}\right\},
$$

where $\kappa_{\pi}$ is the hopping parameter of the pion and $\lambda$ gives the strength of the self-interaction. The total lattice action is the sum of all the above terms, that is

$$
S=S_{\mathrm{N}}+S_{\pi}+S_{\mathrm{NA}}+S_{\mathrm{N} \pi}
$$

\section{$2.1 \quad$ Block fields}

The interaction among hadrons, like nucleons and pions, is non-local as a consequence of the extended hadron structure implied by Quantum Chromodynamics. This non-locality can be approximately taken into account by introducing block fields in the lattice action. Block fields are sums of the the above local fields weighted by appropriate numerical factors. In order to decrease rotation symmetry breaking, our definition of the block fields tries to be as close as possible to an exponential decrease of the weight factors proportional to the Euclidean distance squared. Of course, periodic (or anti-periodic) boundary conditions have to be taken into account and therefore we define the squared distance between two points $x, y$ on the lattice as

$$
(x, y)^{2} \equiv \sum_{\mu=1}^{\mu=4}\left|x_{\mu}, y_{\mu}\right|^{2}
$$

where

$$
\left|x_{\mu}, y_{\mu}\right| \equiv \min \left(\left|x_{\mu}-y_{\mu}\right|,\left|x_{\mu}-y_{\mu}+L_{\mu}\right|,\left|x_{\mu}-y_{\mu}-L_{\mu}\right|\right) .
$$

Here $L_{\mu}$ denotes the lattice extension in the direction $\mu$. With this definition a generic block field is defined by

$$
\Phi_{x} \equiv \sum_{y,(x, y)^{2} \leq R^{2}} \phi_{y} \exp \left\{-S(x, y)^{2}\right\}
$$

The blocking parameters can depend on the type of fields, that is one can have different parameters for the nucleon $\left(R_{\mathrm{N}}, S_{\mathrm{N}}\right)$, for the pion $\left(R_{\pi}, S_{\pi}\right)$ and for the auxiliary fields $\left(R_{0}, S_{0}\right)$ and $\left(R_{1}, S_{1}\right)$, respectively. The lattice action in terms of the block fields has exactly the same form as the above action in terms of the local fields (including the summation over the lattice sites). The only changes are:

$$
\psi_{x} \rightarrow \Psi_{x}, \quad \phi_{x}^{(A)} \rightarrow \Phi_{x}^{(A)} \quad(A=\pi, 0,1) .
$$


Besides reflecting the non-locality of the interactions, the blocking also has an important role in defining the cut-off of the theory. The high momentum modes are decoupled from the interactions by the blocking. Only the modes below some momentum cut-off are interacting, the cut-off value being determined by the blocking parameters $(R, S)$. The cut-off introduced by the blocking can be considered as a physical effect. The lattice cut-off can be changed independently of it. This allows to move the lattice spacing to small values for reducing lattice artefacts.

\section{Numerical simulations}

\subsection{Lattice parameters}

In order to gain experience with the lattice formulation defined in the previous section we started numerical simulations on small lattices and also introduced some simplifications in the choice of lattice action parameters. In most cases we did not block the nucleon field, only the bosonic fields and fixed the blocking parameters as follows:

$$
R_{\mathrm{N}}=0, \quad S_{0}=S_{1}=S_{\pi}=2.0, \quad R_{0}=R_{1}=R_{\pi}=1.5
$$

This choice of the radius sets the number of points in a block to be 33 .

The simplest choice of coupling parameters is to keep only $C_{0}$ and $C_{1}$ defining the fournucleon interactions. We also experimented with the additional introduction of the pion field and the coupling $C_{\pi}$ but here we report only on results in the pion-less theory. A detailed investigation of the effect of the pion coupling is left to future work. Following Ref. [7] $C_{0}$ is taken to be real which corresponds to an attractive interaction whereas $C_{1}=i\left|C_{1}\right|$ is purely imaginary describing a repulsive interaction. In most cases the magnitudes of $C_{0}$ and $C_{1}$ were equal, that is

$$
C_{\pi}=0, \quad C_{0}=\left|C_{0}\right|, \quad C_{1}=i\left|C_{1}\right|, \quad\left|C_{0}\right|=\left|C_{1}\right|
$$

(Note that according to eq. (7) the signs of $C_{0}$ and $C_{1}$ are irrelevant.)

For this exploratory work we had access to a PC cluster with 12 graphics processing units (GPUs) attached to it. Due to the memory limitations of the GPUs the maximally feasible spatial lattice size was $32^{3},(L=32)$. The temporal lattice extension is taken four times longer in order to allow for a precise determination of the energy values. In summary, we did simulations on three types of lattices:

$$
16^{3} \cdot 64, \quad 24^{3} \cdot 96, \quad 32^{3} \cdot 128 .
$$


This restriction to the currently feasible lattice volumes has implications on the choice of parameters we can simulate: in order to study the two nucleon system a large physical volume is required, because otherwise the smallest available lattice momentum is too large. On the other hand we would like to simulate with as small as possible values of the lattice spacing $a$. To set the scale we fixed the equal mass of the proton and neutron to be $M_{\mathrm{N}} \equiv$ $M_{\text {proton }}=M_{\text {neutron }}=939 \mathrm{MeV} / \mathrm{c}^{2}$. This means that, for instance, a nucleon mass in lattice units $a M_{\mathrm{N}}=1$ implies a lattice spacing $a \simeq 0.21 \mathrm{fm}$ and in our cases spatial extensions of $16 a \simeq 3.4 \mathrm{fm}, 24 a \simeq 5.0 \mathrm{fm}$ and $32 a \simeq 6.7 \mathrm{fm}$, respectively. In order to reach larger volumes one can, of course, increase the nucleon mass in lattice units but in this way lattice artefacts will also increase.

With these choices of volumes and with $a M_{\mathrm{N}}=1$ we get for the smallest non-zero momentum $(2 \pi) /(16 L a) \simeq 369 \mathrm{MeV} / \mathrm{c},(2 \pi) /(24 L a) \simeq 246 \mathrm{MeV} / \mathrm{c}$ and $(2 \pi) /(32 L a) \simeq$ $184 \mathrm{MeV} / \mathrm{c}$, respectively. For extracting physics using the approach presented here both lattice sizes and minimal non-zero momenta would require a factor, say, four increase of the lattice extensions which will be comfortably possible to reach with present day computer resources.

\subsection{Numerical methods}

Our present aim is to determine the energies (masses) of different nuclear systems. This can be achieved by investigating the large (Euclidean-) time behaviour of different sets of correlators. Since at present we restrict ourselves to quenched simulations, where the fermion determinant of the nucleon field is neglected, the creation of the boson field configurations is simple. In case of the auxiliary fields $\Phi_{x}^{(0)}$ and $\Phi_{x}^{(1)}$ one has to create Gaussian distributions. The pion field $\Phi_{x}^{(\pi)}$ can also be simply produced by some update algorithm as, for instance, Metropolis algorithm - the only mild complication being to take into account the non-locality introduced by the blocking.

The nucleon mass can be determined from the behaviour of the nucleon time-slice correlator. The time-slice operators are defined as

$$
N_{t} \equiv \sum_{x_{1}, x_{2}, x_{3}}^{x_{4}=t} \Psi_{x_{1}, x_{2}, x_{3}, x_{4}}, \quad \bar{N}_{t} \equiv \sum_{x_{1}, x_{2}, x_{3}}^{x_{4}=t} \bar{\Psi}_{x_{1}, x_{2}, x_{3}, x_{4}}
$$

and the nucleon correlator is, with a Dirac-projection to the state propagating in positive direction,

$$
\operatorname{Tr}_{\text {Dirac }}\left[\left(1+\gamma_{4}\right)\left\langle N_{t_{1}} \bar{N}_{t_{2}}\right\rangle\right] .
$$

The expectation value of the fermion bilinear gives a fermion propagator which is the inverse of the fermion matrix in the fermionic part of the action. The overwhelming part of computer 
resources in our quenched simulations is spent in the calculation of the fermion propagators by an iterative inverter of this sparse matrix.

The computation of nucleon propagators has been done most of the time by applying a mixed precision Conjugate Gradient inverter, see the appendix. The crucial problem for the inverter is to deal with the very small values of the nucleon propagators at large distances. The solution of this problem is to use distance preconditioning following Ref. [25]. Since the nucleon propagator behaves nearly exponentially for distances which we use for extracting the masses (in most cases up to a time distance half the time extension $L_{4}$ of the lattice), we choose the preconditioning function to be

$$
\alpha(t)= \begin{cases}\exp \{-P t\} & \text { if } t \leq L_{4} / 2 \\ \exp \left\{-P\left(L_{4}-t\right)\right\} & \text { if } t>L_{4} / 2\end{cases}
$$

The parameter $P$ can be chosen typically by an amount $0.1-0.5$ smaller than the nucleon mass in lattice units $a m_{\mathrm{N}}$.

In order to obtain the masses of multi-nucleon (in the present paper two-nucleon) states with sufficient precision, one has to find the proper composite operators defining the correlators. Here we restrict ourselves to proton-neutron states. For local operators we take in the spin-0 and spin-1 channels, respectively,

$$
\Psi_{1 x} C \gamma_{5} \Psi_{2 x}, \quad \Psi_{1 x} C \gamma_{k} \Psi_{2 x}, \quad(k=1,2,3)
$$

where $C$ denotes the charge conjugation Dirac matrix.

Especially for scattering states it is important to also take extended (smeared) operators where the proton and neutron are at different points. In case of Gaussian smearing one can use the smearing function

$$
\exp \left\{-\sigma_{1}\left|x_{1}, y_{1}\right|^{2}-\sigma_{2}\left|x_{2}, y_{2}\right|^{2}-\sigma_{3}\left|x_{3}, y_{3}\right|^{2}\right\}
$$

with the notation introduced in (13). For a spherical state in the spin-0 channel one can take $\sigma \equiv \sigma_{1}=\sigma_{2}=\sigma_{3}$. For spin-1, ellipsoidal states with e.g. $\sigma_{1} \neq \sigma_{2}=\sigma_{3}$ are useful. In this latter case we also tried linear smearing corresponding to $\sigma_{2}, \sigma_{3}=\infty$. In order to save computer time one can cut the summation over sites off at distances where the smearing function in (23) is smaller than, say, $10^{-2}$. In case of spherical smearing this corresponds to a cut-off radius of

$$
\rho=\left\{\frac{\log (100)}{\sigma}\right\}^{1 / 2} .
$$

The simplest way to determine the masses is to fit some of the correlators by an exponential function in time intervals for distant time-slices. In case of small enough statistical 
errors one can also obtain good fits with a sum of two (or more) exponentials. The best results can be achieved, however, by taking a set of some operators in a given channel and calculate the correlator matrix among them. For determining the energies of two-nucleon (actually proton-neutron) states we typically start from a $4 \times 4$ correlator matrix. The four states are chosen from local, spherically smeared and elliptically smeared states with different Dirac-matrices.

The correlator matrix can be approximated by the sum of contributions of eigenstates of the Hamiltonian (i. e. of the transfer matrix). In general, a real symmetric $D \times D$ correlator matrix $C\left(t_{2}, t_{1}\right)$ between time-slices $t_{1}$ and $t_{2}>t_{1}$ is defined by the matrix elements of $D$ operators $\mathcal{O}_{a}, \mathcal{O}_{b}, \ldots, \mathcal{O}_{d}$. If the energy eigenstates are $|n\rangle, n=1,2, \ldots, M$ then in a shorthand notation

$$
C\left(t_{2}, t_{1}\right)=\left(\begin{array}{cccc}
C\left(t_{2}, t_{1}\right)_{a a} & C\left(t_{2}, t_{1}\right)_{a b} & \ldots & C\left(t_{2}, t_{1}\right)_{a d} \\
C\left(t_{2}, t_{1}\right)_{a b} & C\left(t_{2}, t_{1}\right)_{b b} & \ldots & C\left(t_{2}, t_{1}\right)_{b d} \\
\vdots & \vdots & \ldots & \vdots \\
C\left(t_{2}, t_{1}\right)_{a d} & C\left(t_{2}, t_{1}\right)_{b d} & \ldots & C\left(t_{2}, t_{1}\right)_{d d}
\end{array}\right)
$$

where the matrix elements can be written as, for instance,

$$
C\left(t_{2}, t_{1}\right)_{a b}=(a \mid 1)_{t_{2}}(b \mid 1)_{t_{1}}+(a \mid 2)_{t_{2}}(b \mid 2)_{t_{1}}+\ldots+(a \mid M)_{t_{2}}(b \mid M)_{t_{1}}
$$

with

$$
(c \mid k)_{t} \equiv\left\langle 0\left|\mathcal{O}_{c}(t)\right| k\right\rangle=\left\langle k\left|\mathcal{O}_{c}(t)\right| 0\right\rangle
$$

for $c=a, b, \ldots, d$ and $k=1,2, \ldots, M$.

Assuming that we consider bosonic (fermionic) operators, we have periodic (anti-periodic) time dependence with the time extension of the lattice $L_{4}$. This implies

$$
(a \mid k)_{t_{2}}(b \mid k)_{t_{1}}=(a \mid k)(b \mid k)\left\{\exp \left[-t E_{k}\right] \pm \exp \left[-\left(L_{4}-t\right) E_{k}\right]\right\}
$$

where the positive and negative sign stands for periodicity and anti-periodicity, respectively. Here $t \equiv t_{2}-t_{1}, E_{k}$ is the energy (e.g. mass) corresponding to the state $|k\rangle$ and $(a \mid k) \equiv(a \mid k)_{0},(b \mid k) \equiv(b \mid k)_{0}$. Fitting the correlator matrix by the expression given by (25) - (28) one can obtain the energies we are looking for [26]. The statistical errors of the results can also be obtained by methods similar to those described in Section 5 of this reference.

Since in the present case the relevant (multi-) nucleon correlators can be determined to a very good precision, one can perform least-square fits by minimising the correlated chisquared. In order to obtain a good starting point for the minimisation, one can first minimise the uncorrelated chi-squared defined by

$$
\chi_{n}^{2}=\sum_{i=1}^{N_{C}}\left(\frac{f_{i}(p)-\bar{X}_{i}}{\delta X_{i}}\right)^{2}
$$


where the index $i$ runs over the independent matrix elements to be fitted, $\bar{X}_{i}$ and $\delta X_{i}$ are the mean value and error of the matrix element $i$, respectively, and $f_{i}(p)$ is the fitting function of $N_{P}$ parameters $\left(p_{1}, p_{2}, \ldots, p_{N_{P}}\right)$ defined by (26)-(28). The best fit obtained in this way can be taken as a starting point to minimise the correlated chi-squared

$$
\chi_{c}^{2}=\sum_{i, j=1}^{N_{C}}\left(f_{i}(p)-\bar{X}_{i}\right) M_{i j}\left(f_{j}(p)-\bar{X}_{j}\right),
$$

where $M_{i j}=N C_{i j}^{-1}$, with the number $N$ of input data and the correlator matrix

$$
C_{i j}=\frac{1}{N-1} \sum_{n=1}^{N}\left(X_{i, n}-\bar{X}_{i}\right)\left(X_{j, n}-\bar{X}_{j}\right) .
$$

In general, the correlator matrix in (31) can be determined with sufficient precision for obtaining its inverse and its eigenvectors. In some cases, in particular if the dimension of the correlator matrix $N_{C}$ is large, smoothing of the smallest eigenvalues [27,28] can be helpful but does not substantially influence the results. The advantage of properly obtaining the minimum of $\chi_{c}^{2}$ is that one can select "good fits" by the value of $\chi_{c}^{2}$ per number of degrees of freedom $\left(N_{C}-N_{P}\right)$. The mean value and error of a quantity is defined by considering the distribution of its values in good fits. The quoted value is then the position of the median of the distribution of these selected values. The error defines a (symmetric) interval around the median such that $68 \%$ of the distribution is contained in it.

\subsection{Numerical results}

The physical quantities we are interested in are for instance the nucleon-nucleon scattering length and binding energies of multi nucleon states. In a lattice simulation, the determination of these quantities requires a study of the (finite) volume dependence of one and two (and multiple) particle energies. In this methodical paper we hence try to understand how precisely the corresponding quantities, i.e. the nucleon and two-nucleon masses, can be determined.

In order to do so, we performed several simulations and determined the masses as described in the previous sub-section. A typical example is a run on a $32^{3} \cdot 128$ lattice at $\kappa_{\mathrm{N}}=0.08, C_{0}=0.2, C_{1}=0.2 i$. It turned out that the masses can be very precisely obtained even from a modest statistical sample of 120 configurations: see figures 1, 3 ,

In figure 1 we plot the actual nucleon and two nucleon correlators as functions of the time $t$ in lattice units on a logarithmic scale. The decay is nearly exponential in the whole time range. Fitting the correlators in different time-slice distance intervals $\left[t_{1}, t_{2}\right]$ by minimising the correlated chi-squared one finds that for the nucleon mass $a m_{\mathrm{N}}$ we observe a plateau 


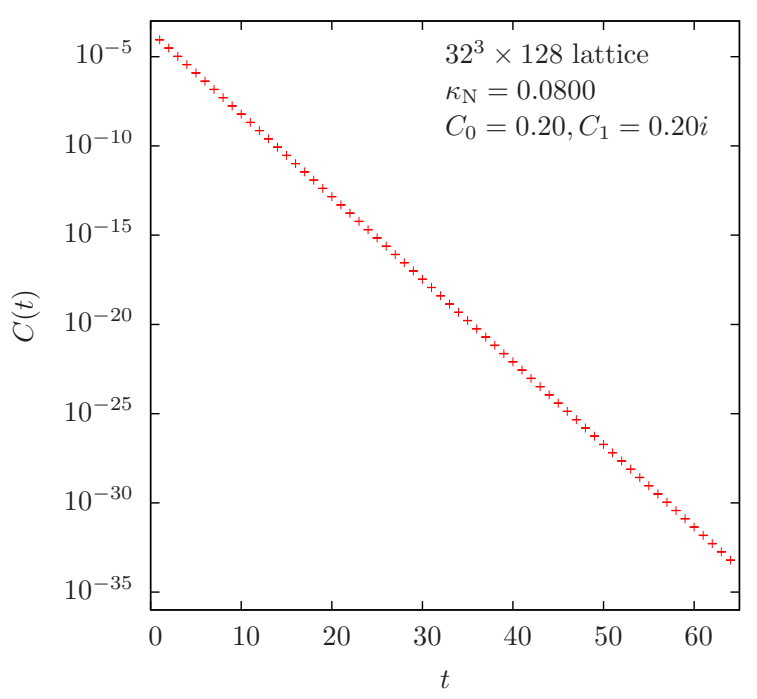

(a)

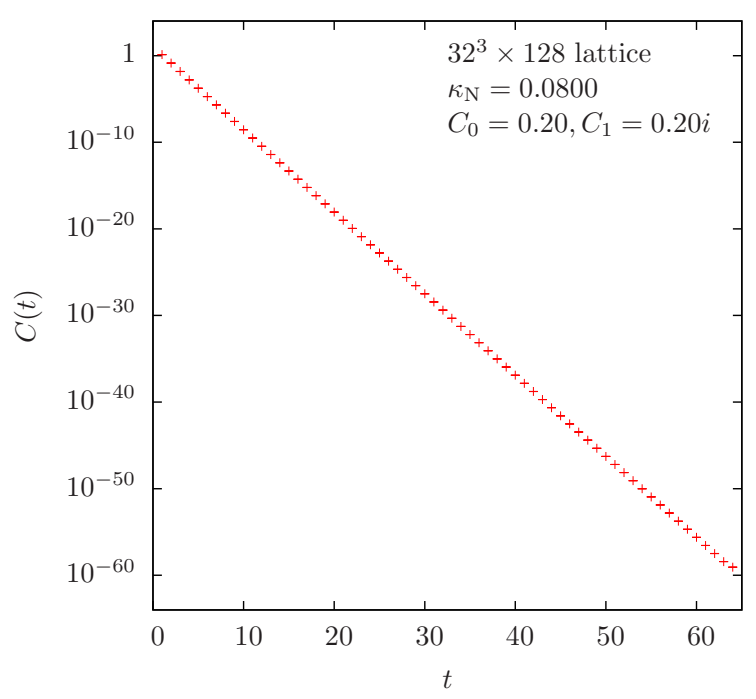

(b)

Figure 1: The nucleon correlator (a) and two-nucleon correlator (b) on a logarithmic scale as a function of time-slice distance $t$ in lattice units on a $32^{3} \cdot 128$ lattice at $\kappa_{\mathrm{N}}=0.08, C_{0}=$ $-i C_{1}=0.2$.

from $t_{1}=18$ on, almost independent of the value of $t_{2}$ (see fig. 2). For the two nucleon mass $a m_{\mathrm{NN}}$ the plateau sets in somewhat later (see fig. 3). Both quantities have in common that the statistical errors of the single points are in the sub-percent region. The mass value and its error as determined from the distribution of the fit results - as described earlier - are indicated in both plots by the horizontal lines.

The required precision of two-nucleon energies can be exemplified by Lüscher's formula for extracting scattering lengths from finite size effects of the two-particle energies [29]. To leading order one can express the scattering length $a_{0}$ as

$$
a_{0} m_{\mathrm{N}}=\left(2-\frac{m_{\mathrm{NN}}}{m_{\mathrm{N}}}\right) \cdot \frac{\left(m_{\mathrm{N}} L a\right)^{3}}{4 \pi} .
$$

The masses from Figures 2, 23, namely $a m_{\mathrm{N}}=1.06567(25)$ and $a m_{\mathrm{NN}}=2.1511(20)$ give for the right hand side a value $-59(7)$. The physical value of the right hand side is $a_{0} m_{\mathrm{N}}=113.1$. This corresponds to the value of the scattering length in the ${ }^{1} S_{0}$ channel $a_{0}=+23.76 \mathrm{fm}$ and $m_{\mathrm{N}}=939 \mathrm{MeV}$. (Here we use the sign convention for the scattering length of Ref. [29].1.) Our value has the right order of magnitude but an opposite sign which is due to the strong repulsion implied by the imaginary value $C_{1}=0.2 i$. Obviously, tuning to the physical value

\footnotetext{
${ }^{1}$ We thank the referee of our paper for drawing our attention to the different sign conventions of the scattering length in the literature which we overlooked.
} 


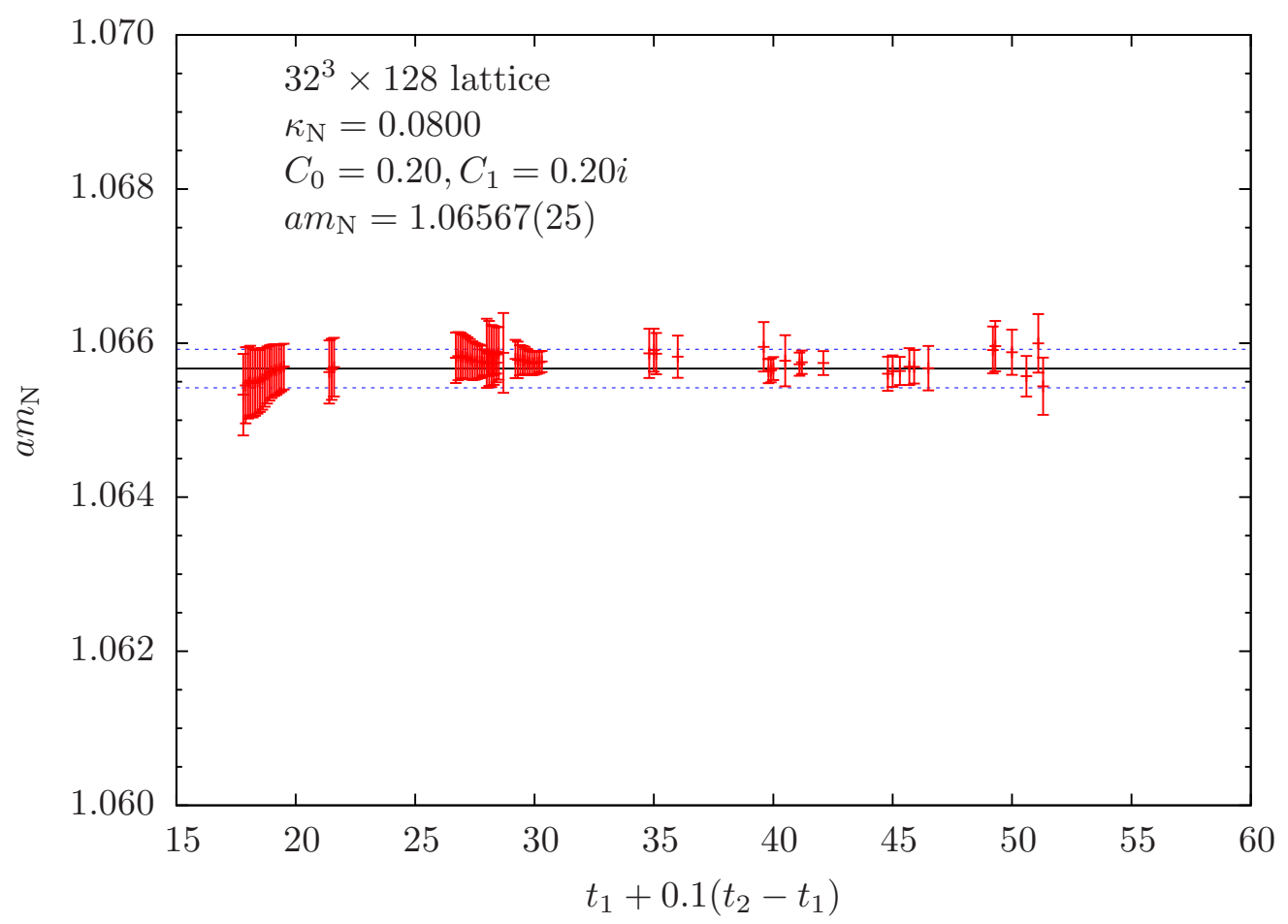

Figure 2: Results of nucleon mass am $m_{\mathrm{N}}$ fits on different fitting intervals for a $32^{3} \cdot 128$ lattice at $\kappa_{N}=0.08, C_{0}=-i C_{1}=0.2$. The fit interval $\left[t_{1}, t_{2}\right]$ is specified on the $x$-axis by $t_{1}+0.1\left(t_{2}-t_{1}\right)$. The horizontal lines indicate the final result and error obtained from the distribution of the fit results.

would require either a much smaller imaginary value or even a real value of $C_{1}$. Besides of this, one cannot assume that the asymptotic formula works well already on a volume of extension $\simeq 7 \mathrm{fm}$. In fact, on lattices $16^{3} \cdot 64$ and $24^{3} \cdot 96$ in the same point we obtained for the right hand side of (32) values of about 5 and 40, respectively. This shows that, in any case, for the determination of scattering lengths simulations on larger volumes are required.

\section{Outlook}

In this paper we have defined a theory of nucleon and pion fields in the Euclidean path integral formulation on the lattice. The inherent physical cut-off of this theory has been implemented by formulating the lattice action in terms of blocked fields. This physical cutoff is given by the block size. In this way the lattice cut-off is separated from the physical cut-off and can be changed in order to determine the size of lattice artefacts.

The positive outcome of the first studies we have performed is that the correlators of single- and two-nucleon systems can be determined very precisely in order to obtain the 


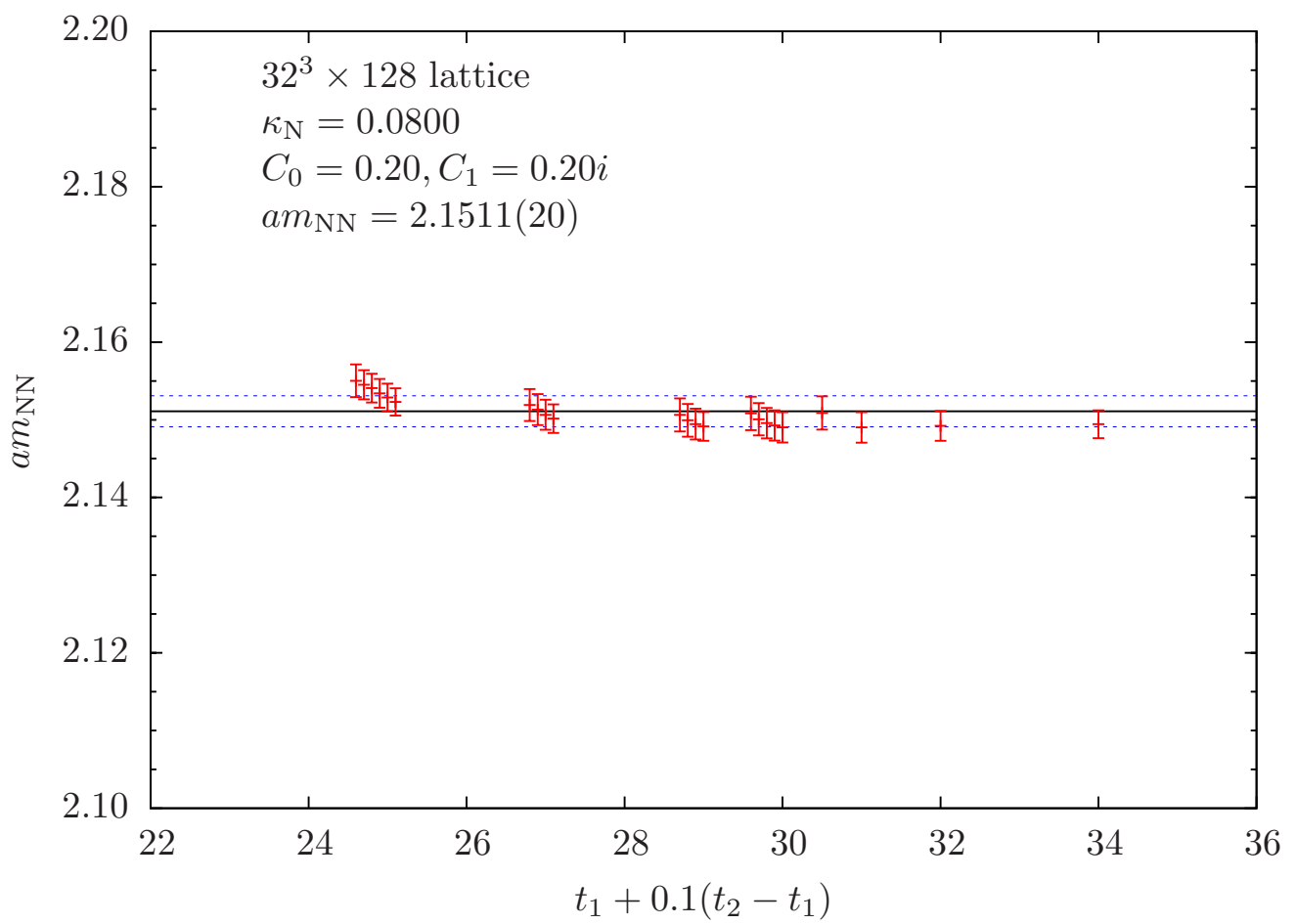

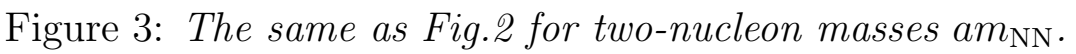

energies to a very good precision. In fact a few thousands of configurations are sufficient for a precision better than one per mill. Using Lüscher's formula we could determine values for the nucleon-nucleon scattering length. The corresponding results are positive in the sense that with larger volumes it seems to be realistic to tune its value to the physical one.

An important step towards obtaining physical results is hence to increase the physical lattice sizes. As discussed in Section 3.1, a spatial lattice extension of about $L=100$ would correspond for a nucleon mass in lattice units $a M_{\mathrm{N}} \simeq 1$ to a lattice size of about $L a \simeq 20 \mathrm{fm}$ and a minimal lattice momentum $2 \pi /(100 L a) \simeq 60 \mathrm{MeV} / \mathrm{c}$.

It will be also important to introduce the pion field besides the auxiliary fields describing four-nucleon couplings.

In order to complete this sort of lattice studies an important final step is to investigate the dependence of the results on the lattice spacing. For this the physical parameters as, for instance, the block size parameters $R m_{\mathrm{N}}, S m_{\mathrm{N}}$ and the lattice volume $L a m_{\mathrm{N}}$ have to be kept fixed. The couplings in the lattice action $\left(C_{0,1}\right.$ etc.) have to be tuned for each value of the lattice spacing in such a way that some well chosen physical quantities (as for instance some nucleon phase shifts) take their physical values. Of course, if the lattice spacing gets smaller the required number of lattice points have to be increased correspondingly and this implies an increase in the required computational power. 
A possible source of difficulties in the quenched approximation, as observed in Yukawa models by the authors of [19 21], is the appearance of exceptional configurations with extremely small eigenvalues of the fermion lattice action. These configurations make the determination of correlators and therefore masses practically impossible. In our case we found exceptional configurations for bare couplings in the range $\left|C_{0}\right|,\left|C_{1}\right|,\left|C_{\pi}\right|>0.3$. Since this problem does not appear in numerical simulations in Yukawa models with dynamical fermions [22,23], we expect that it does also disappear in our nuclear Yukawa models if dynamical nucleons are included in the simulation update. For real values of the couplings $C_{0}, C_{1}, C_{\pi}$ the fermion determinant is real (non-negative) therefore the known Hybrid Monte Carlo methods [30] can be applied in a straightforward manner. For non-real (e.g. imaginary) couplings the determinant becomes complex and the numerical simulation turns non-trivial, if not impossible.

\section{Acknowledgement}

We thank Ulf G. Meißner for introducing us in the literature of nuclear physics on the lattice. We thank Hans-Werner Hammer, Dean Lee and Ulf G. Meißner for helpful and interesting discussions. We are grateful to Hans-Werner Hammer for useful comments on the manuscript. We thank JSC at FZ-Jülich for providing computing time on JUROPA.

\section{A Implementation Details}

As mentioned in the introduction, we have used graphics processing units (GPUs) in order to perform the numerical inversions of the Dirac operator. We have 12 NVIDIA Tesla C1060 GPUs available with four Gb of memory each. We have used NVIDIAs CUDA environment to implement the Dirac operator deduced from eqs. (6), (8) and (9) for GPUs, which is very similar to available implementations for lattice QCD, see for instance ref. [31].

We employ a mixed precision solver using both, the CPU and the GPU. On the GPU we have implemented a conjugate gradient $(\mathrm{CG})$ solver inverting the squared hermitian Dirac operator (since $C_{1}$ is purely imaginary)

$$
Q^{\dagger} Q=\gamma_{5} D\left(C_{0},-C_{1}, \kappa_{N}\right) \gamma_{5} D\left(C_{0}, C_{1}, \kappa_{N}\right)
$$

The desired result is then obtained by multiplying with $Q^{\dagger}$. The CG solver on the GPU is implemented solely in single precision (32 Bit). The CG solver is called from an outer solver, which is run on the CPU in double precision (64 Bit). We use iterative refinement as the outer solver in order to solve

$$
D \eta=\phi
$$




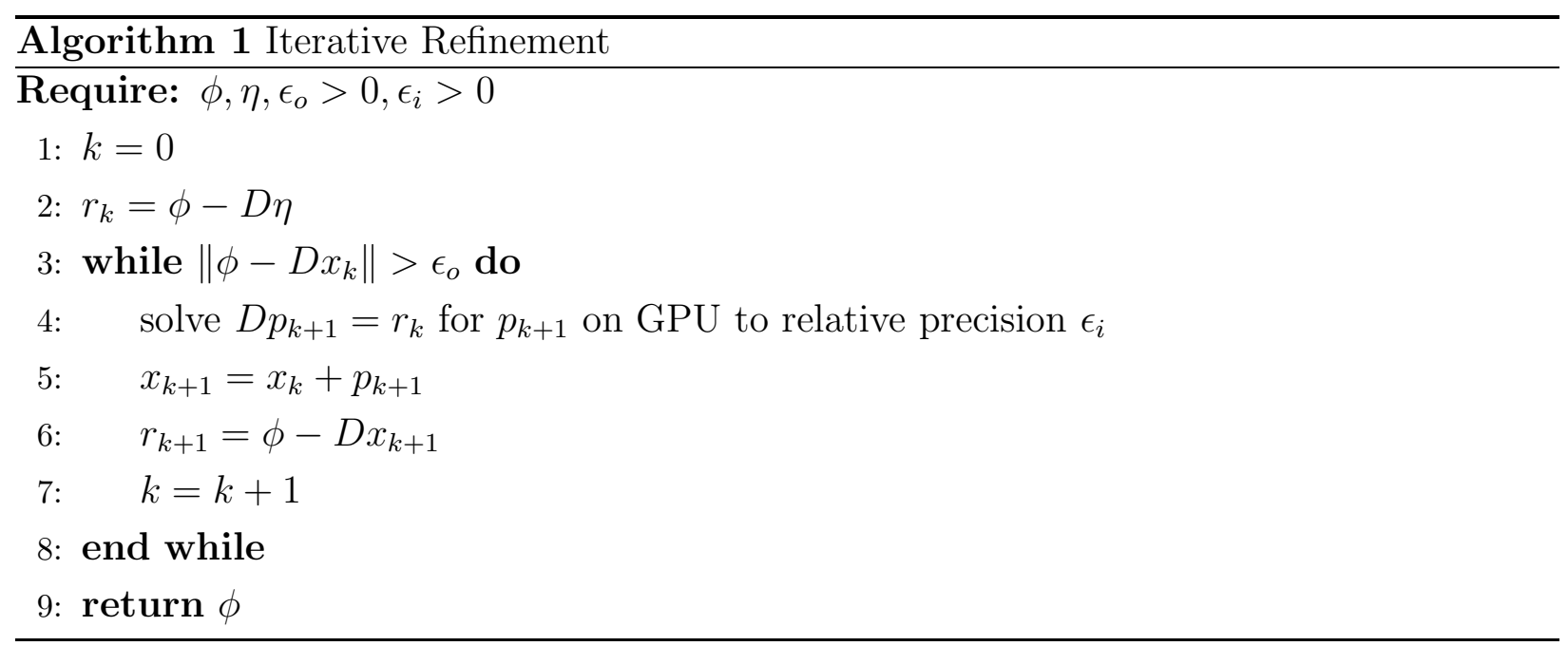

for $\eta$, given some source spinor field $\phi$. The algorithm is summarised in algorithm 1. Depending on the parameters $\kappa_{N}, C_{0}$ and $C_{1}$ one has to tune the precision for which to solve on the GPU. The usage of distance preconditioning also had significant influence on this tuning: the closer the preconditioning mass to the measured mass the less stable the prescribed mixed precision solver turned out to behave. Most probably due to accumulation of round off errors we had to reduce the number of inner iterations further and further with preconditioning mass approaching the measured mass. 


\section{References}

[1] S. Weinberg, Phys.Lett. B251, 288 (1990).

[2] S. Weinberg, Nucl.Phys. B363, 3 (1991).

[3] D. Lee, Prog.Part.Nucl.Phys. 63, 117 (2009), arXiv:0804.3501 [nucl-th].

[4] E. Epelbaum, H.-W. Hammer and U.-G. Meißner, Rev.Mod.Phys. 81, 1773 (2009), arXiv:0811.1338 [nucl-th].

[5] R. Machleidt and D. Entem, Phys.Rept. 503, 1 (2011), arXiv:1105.2919 [nucl-th].

[6] B. Borasoy, H. Krebs, D. Lee and U. G. Meißner, Nucl. Phys. A768, 179 (2006), arXiv:nucl-th/0510047.

[7] B. Borasoy, E. Epelbaum, H. Krebs, D. Lee and U.-G. Meißner, Eur. Phys. J. A31, 105 (2007), arXiv:nucl-th/0611087.

[8] B. Borasoy, E. Epelbaum, H. Krebs, D. Lee and U.-G. Meißner, Eur. Phys. J. A35, 343 (2008), arXiv:0712.2990 [nucl-th].

[9] E. Epelbaum, H. Krebs, D. Lee and U.-G. Meißner, Eur. Phys. J. A41, 125 (2009), arXiv:0903.1666 [nucl-th].

[10] E. Epelbaum, H. Krebs, D. Lee and U.-G. Meißner, Phys. Rev. Lett. 104, 142501 (2010), arXiv:0912.4195 [nucl-th].

[11] E. Epelbaum, H. Krebs, D. Lee and U.-G. Meißner, Eur. Phys. J. A45, 335 (2010), arXiv: 1003.5697 [nucl-th].

[12] E. Epelbaum, H. Krebs, D. Lee and U.-G. Meißner, Phys.Rev.Lett. 106, 192501 (2011), arXiv:1101.2547 [nucl-th].

[13] D. Lee and T. Schäfer, Phys. Rev. C72, 024006 (2005), arXiv:nucl-th/0412002.

[14] Quantum Fields on a Lattice (Cambridge University Press, 1994).

[15] K. Symanzik, Presented at 6th Int. Conf. on Mathematical Physics, Berlin, West Germany, Aug 11-21, 1981.

[16] K. Symanzik, Nucl. Phys. B226, 187 (1983).

[17] K. Symanzik, Nucl. Phys. B226, 205 (1983). 
[18] M. Lüscher, S. Sint, R. Sommer and P. Weisz, Nucl. Phys. B478, 365 (1996), arXiv:hep-lat/9605038.

[19] F. De Soto et al., Eur. Phys. J. A31, 777 (2007), arXiv:hep-lat/0610084.

[20] F. De Soto et al., Nucl. Phys. A790, 410 (2007), arXiv:hep-lat/0610086.

[21] F. de Soto, J. d'Auriac and J. Carbonell, arXiv:1104.1907 [hep-lat].

[22] K. Farakos et al., Nucl.Phys. B350, 474 (1991).

[23] L. Lin, I. Montvay, G. Münster, M. Plagge and H. Wittig, Phys.Lett. B317, 143 (1993), arXiv:hep-lat/9303012 [hep-lat].

[24] K. G. Wilson, Phys. Rev. D10, 2445 (1974).

[25] G. M. de Divitiis, R. Petronzio and N. Tantalo, Phys. Lett. B692, 157 (2010), arXiv:1006.4028 [hep-lat].

[26] European Twisted Mass Collaboration, R. Baron et al., Comput. Phys. Commun. 182, 299 (2011), arXiv:1005.2042 [hep-lat].

[27] C. Michael, Phys. Rev. D49, 2616 (1994), arXiv:hep-lat/9310026.

[28] C. Michael and A. McKerrell, Phys. Rev. D51, 3745 (1995), arXiv:hep-lat/9412087.

[29] M. Lüscher, Commun.Math.Phys. 105, 153 (1986).

[30] S. Duane, A. D. Kennedy, B. J. Pendleton and D. Roweth, Phys. Lett. B195, 216 (1987).

[31] M. Clark, R. Babich, K. Barros, R. Brower and C. Rebbi, Comput.Phys.Commun. 181, 1517 (2010), arXiv:0911.3191 [hep-lat]. 